\title{
Upregulation of proline rich 11 is an independent unfavorable prognostic factor for survival of tongue squamous cell carcinoma patients
}

\author{
CHUNYANG WANG ${ }^{1 *}$, LIANG YU ${ }^{2 *}$, FENGCHUN HU $^{3}$, JUAN WANG ${ }^{1}$, \\ XIJUAN CHEN $^{1}$, SHANSHAN TAI ${ }^{1}$ and BIN CHENG ${ }^{1}$
}

\begin{abstract}
${ }^{1}$ Guangdong Provincial Key Laboratory of Stomatology, Guanghua School of Stomatology, Hospital of Stomatology, Sun Yat-sen University, Guangzhou, Guangdong 510055; ${ }^{2}$ Department of Thyroid and Breast Surgery,

The First Affiliated Hospital of Sun Yat-sen University, Guangzhou, Guangdong 510080; ${ }^{3}$ Department of Stomatology, The First Affiliated Hospital of Guangzhou University of Chinese Medicine, Guangzhou, Guangdong 510405, P.R. China
\end{abstract}

Received January 21, 2016; Accepted May 24, 2017

DOI: $10.3892 / 01.2017 .6780$

\begin{abstract}
Proline rich 11 (PRR11) serves an important role in the development and progression of a number of types of human cancer. However, the clinical role of PRR11 in tongue squamous cell carcinoma (TSCC) remains unknown. The present study aimed to investigate the expression and clinicopathological significance of PRR11 in TSCC. The Cancer Genome Atlas analysis demonstrated that the upregulation of PRR11 in TSCC correlated with poor prognosis. The data of the present study revealed that PRR11 mRNA and protein expression was markedly upregulated in human TSCC tissues. Immunohistochemistry on 72 archived paraffin-embedded TSCC specimens suggested that high levels of PRR11 expression were significantly associated with clinical stage $(\mathrm{P}<0.001)$, T classification $(\mathrm{P}=0.009), \mathrm{N}$ classification $(\mathrm{P}=0.017)$ and vital status $(\mathrm{P}=0.010)$. In addition, patients with TSCC with higher PRR11 expression exhibited substantially shorter survival times compared with patients with lower PRR11 expression $(\mathrm{P}<0.001)$. Univariate and multivariate analyses indicated that PRR11 upregulation may be an independent prognostic factor for patients with TSCC $(\mathrm{P}=0.001)$. Taken together, and to the best of our knowledge, the results of the present study demonstrated for the first time that PRR11 is involved in the
\end{abstract}

Correspondence to: Professor Bin Cheng, Guangdong Provincial Key Laboratory of Stomatology, Guanghua School of Stomatology, Hospital of Stomatology, Sun Yat-sen University, 56 Lingyuan Xi Road, Guangzhou, Guangdong 510055, P.R. China

E-mail: chengbin@mail.sysu.edu.cn

*Contributed equally

Abbreviations: PRR11, proline rich 11; TSCC, tongue squamous cell carcinoma

Key words: proline rich 11, tongue squamous cell carcinoma, prognosis, immunohistochemistry development and progression of TSCC, and may serve as a useful prognostic marker and an effective target for treating TSCC.

\section{Introduction}

Tongue squamous cell carcinoma (TSCC) is the most commonly occurring type of oral cancer (1). Due to the high risk of occult metastasis and neck nodal metastasis, patients with TSCC exhibit a significantly poorer prognosis compared with those with other cancers of the oral cavity (2). The prognosis of TSCC remains reliant on the Tumor Node Metastasis (TNM) staging (3) of the tumor; however, the outcome of patients at the same stage may vary considerably. Thus, novel prognostic indicators are required.

Proline rich 11 (PRR11) was first identified during a screen for novel cancer-associated genes (4). PRR11 is a 360-amino acid protein that is encoded by a gene located on human chromosome 17q22 (5). Human chromosome 17 hosts a number of other cancer-associated genes, including the essential tumor suppressor genes tumor protein $(p) 53$ and breast cancer 1 $(6,7)$. PRR11 comprises 10 exons, and the encoded protein typically serves as a ligand for SRC Homology 3 (SH3), WW and enabled/VASP homology 1 domains (8). PRR11 expression is elevated in lung and breast cancer, and numerous types of tumors of the digestive system $(5,8,9)$. PRR11 is also suggested to be associated with tumor development and progression (5,8-10). However, whether PRR11 is involved in tongue squamous cell carcinoma has not been determined. The present study aimed to investigate the expression of PRR11 in tongue squamous cell carcinoma, and to examine its association with clinical parameters and prognosis in patients with TSCC.

\section{Materials and methods}

The Cancer Genome Atlas (TCGA) TSCC data mining. PRR11 mRNA expression data from 126 TSCC and 12 non-cancerous tongue tissue samples were downloaded from the TCGA 
database (http://cancergenome.nih.gov/) in December 2014, along with overall patient survival data. The association between PRR11 expression and overall survival was evaluated by comparing the top, and bottom $50 \%$ of the specimens, using the log-rank test.

Tissue specimens and patient information. Fresh tumor specimens were collected from patients with TSCC who had undergone surgery at The First Affiliated Hospital of Sun Yat-sen University (Guangzhou, China) from March 2014 to October 2014 and used for quantitative reverse transcription polymerase chain reaction (RT-qPCR) and western blotting. For immunohistochemistry, 72 TSCC paraffin-embedded specimens were prepared at The First Affiliated Hospital of Sun Yat-sen University between January 2007 and September 2010 from patients who were histopathologically, and clinically diagnosed with TSCC. The male: female ratio of the patients included in the present study was 38:34, and the median age was 54 (age range, 28-80 years). No patients received any additional therapy prior to surgery. Patients with apparent distant metastasis were excluded. Tumor grade and stage were defined according to the 6th edition of the TNM classification of the Union for International Cancer Control (UICC, 2002) (3). Written informed consent and approval from the First Affiliated Hospital of Sun Yat-sen University Institutional Review Board were obtained from all participants prior to any experiments. Sample clinical information is summarized in Table I.

$R T-q P C R$. Total RNA was isolated using TRIzol reagent (Invitrogen; Thermo Fisher Scientific, Inc., Waltham, MA, USA) according to the manufacturer's protocol. The concentration and quality of RNA were measured spectrophotometrically at 260 , and $280 \mathrm{~nm}$. RNA was reverse-transcribed by heating at $25^{\circ} \mathrm{C}$ for $10 \mathrm{~min}$, then at $55^{\circ} \mathrm{C}$ for $30 \mathrm{~min}$, and at $85^{\circ} \mathrm{C}$ for 5 min to produce cDNA using the Oligo (dT) 15 primer and M-MLV Reverse Transcriptase kit (Promega Corporation, Madison, WI, USA). Primers were as follows: PRR11 forward, 5'-GACTTCCAAAGCTGTGCTTCC-3' and reverse, 5'-CTG CATGGGTCCATCCTTTTT-3'; 18S rRNA forward, 5'-CCT GGATACCGCAGCTAGGA-3' and reverse, 5'-GCGGCGCAA TACGAATGCCCC-3'. qPCR was performed with FastStart Universal SYBR Green Master (Rox; Roche Diagnostics $\mathrm{GmbH}$, Mannheim, Germany) as follows: $2 \mathrm{~min}$ at $95^{\circ} \mathrm{C}$; followed by 40 cycles of $10 \mathrm{sec}$ at $95^{\circ} \mathrm{C}, 30 \mathrm{sec}$ at $60^{\circ} \mathrm{C}$, and $30 \mathrm{sec}$ at $72^{\circ} \mathrm{C}$. qPCR was performed using the ABI Prism 7900 HT real-time PCR system (Applied Biosystems; Thermo Fisher Scientific, Inc.). The $2^{-\Delta \Delta \mathrm{Cq}}$ method was used to calculate gene expression relative to the 18SrRNA housekeeping control (11). All experiments were repeated in triplicate.

Western blotting. Fresh tissue samples were ground to powder in liquid nitrogen and lysed with 10 times the tissue volume of the pre-cooled radioimmunoprecipitation assay buffer (Wuhan Boster Biological Technology, Ltd., Wuhan, China) containing phosphatase inhibitors (Phosphatase Inhibitor Cocktails Set II, Calbiochem; Merck KGaA, Darmstadt, Germany), proteinase inhibitors (Protease Inhibitor Cocktails Set I, Calbiochem; Merck KGaA) and $1 \mathrm{mmol} / \mathrm{l}$ phenylmethanesulfonyl fluoride (Sigma-Aldrich; Merck KGaA). Lysate protein concentration was measured by bicinchoninic protein
Table I. Clinicopathological characteristics and proline rich 11 expression in patients tongue squamous cell carcinoma.

\begin{tabular}{|c|c|}
\hline Variable & Number of cases $(\%)$ \\
\hline \multicolumn{2}{|l|}{ Sex } \\
\hline Male & $38(52.8)$ \\
\hline Female & $34(47.2)$ \\
\hline \multicolumn{2}{|l|}{ Age, years } \\
\hline$\geq 54$ & $36(50.0)$ \\
\hline$<54$ & $36(50.0)$ \\
\hline \multicolumn{2}{|l|}{ Clinical stage } \\
\hline I & $11(15.3)$ \\
\hline II & $27(37.5)$ \\
\hline III & $25(34.7)$ \\
\hline IV & $9(12.5)$ \\
\hline \multicolumn{2}{|l|}{ T classification } \\
\hline $\mathrm{T} 1$ & $16(22.2)$ \\
\hline $\mathrm{T} 2$ & $46(63.9)$ \\
\hline T3 & $7(9.7)$ \\
\hline $\mathrm{T} 4$ & $3(4.2)$ \\
\hline \multicolumn{2}{|l|}{$\mathrm{N}$ classification } \\
\hline N0 & $46(63.9)$ \\
\hline N1 & $19(26.4)$ \\
\hline $\mathrm{N} 2$ & $7(9.7)$ \\
\hline \multicolumn{2}{|l|}{ M classification } \\
\hline No & $72(100.0)$ \\
\hline Yes & $0(0.0)$ \\
\hline \multicolumn{2}{|c|}{ Differentiation grade } \\
\hline Well & $40(55.6)$ \\
\hline Moderate & $26(36.1)$ \\
\hline Poor & $6(8.3)$ \\
\hline \multicolumn{2}{|c|}{ Vital status (at follow-up) } \\
\hline Alive & $42(58.3)$ \\
\hline Succumbed & $30(41.7)$ \\
\hline \multicolumn{2}{|c|}{ Expression of PRR11 } \\
\hline Low & $27(37.5)$ \\
\hline High & $45(62.5)$ \\
\hline Detectable & $71(98.6)$ \\
\hline Undetectable & $1(1.4)$ \\
\hline
\end{tabular}

$\mathrm{T}$, tumor; $\mathrm{N}$, node; $\mathrm{M}$, metastasis.

assay (Sigma-Aldrich; Merck KGaA). Proteins (40 $\mu \mathrm{g} / \mathrm{lane})$ were separated by $10 \%$ SDS-PAGE and transferred to a polyvinylidene fluoride membrane (EMD Millipore, Billerica, MA, USA). To avoid unspecific binding, the membrane was blocked with $5 \%$ non-fat milk (Merck $\mathrm{KGaA}$ ) in phosphate buffered saline (PBS)/Tween $(0.05 \%)$ at room temperature for $1 \mathrm{~h}$. Subsequently, the membrane was incubated with a polyclonal rabbit anti-human PRR11 antibody (dilution, 1:250; cat. no. NBP1-83784; Novus Biologicals, LLC, Littleton, CO, USA) overnight at $4^{\circ} \mathrm{C}$, and anti- $\beta$-actin monoclonal antibody (dilution, 1:1,000; cat. no. ab8226, Abcam Inc., Cambridge, MA, 
A

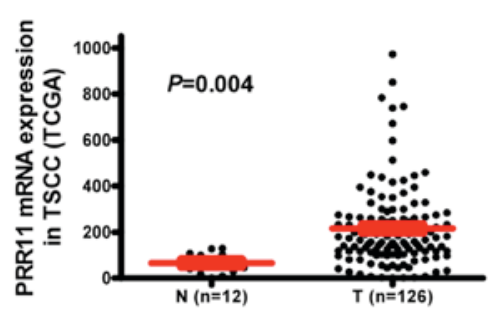

C

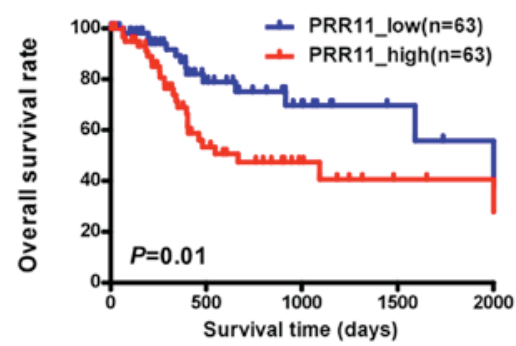

B

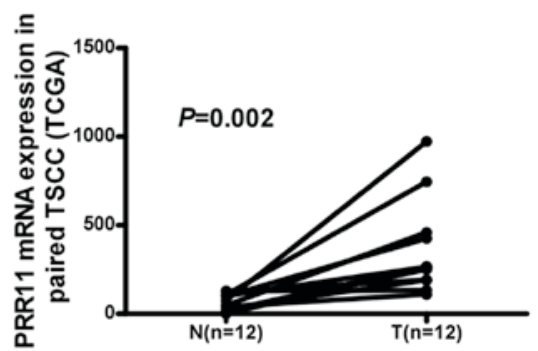

Figure 1. PRR11 overexpression in TSCC specimens from TCGA data. (A) PRR11 mRNA levels in 126 TSCC biopsies and 12 non-cancerous tongue tissues. (B) PRR11 mRNA levels in 12 pairs of TSCC tissues (T) and equivalent non-cancerous tissues (ANT) from the same patient. (C) Kaplan-Meier survival analysis with log-rank test for overall survival in all 126 patients with TSCC. PRR11, proline rich 11; TSCC, tongue squamous cell carcinoma; TCGA, The Cancer Genome Atlas.

UK) was used as the loading control, and then incubated with a horseradish peroxidase-conjugated affiniPure goat anti-rabbit secondary antibody (dilution, 1:10,000; cat. no. 111-035-003, Jackson ImmunoResearch Inc., West Grove, PA, USA) at room temperature for $1 \mathrm{~h}$. Immunoreactive bands were visualized with an enhanced chemiluminescence detection system (EMD Millipore, Billerica, MA, USA).

Immunohistochemistry (IHC). IHC was performed on 72 human TSCC tissues. Antigen retrieval was performed by heating these sections in $10 \mathrm{mmol} / 1$ citric acid buffer $(\mathrm{pH} 6.0)$. The sections were blocked with 5\% normal goat serum (Wuhan Boster Biological Technology, Ltd.) for $30 \mathrm{~min}$ at $25^{\circ} \mathrm{C}$, and incubated in $3 \%$ hydrogen peroxide at $25^{\circ} \mathrm{C}$. Sections were then incubated with a polyclonal rabbit anti-human PRR11 antibody $(1: 100)$ at $4^{\circ} \mathrm{C}$ overnight, followed by incubation with a horseradish peroxidase-conjugated goat anti-rabbit secondary antibody (dilution, 1:1,000; cat. no. 111-035-003, Jackson ImmunoResearch Laboratories Inc., West Grove, PA, USA) for $1 \mathrm{~h}$ at $25^{\circ} \mathrm{C}$. Finally, slides were treated with chromogen 3,3'-diaminobenzidine (Dako; Agilent Technologies, Inc., Santa Clara, CA, USA) for $1 \mathrm{~min}$ and counterstained with $5 \%$ hematoxylin for $20 \mathrm{sec}$ at $25^{\circ} \mathrm{C}$. The degree of immunostaining of the sections was viewed and scored separately by two independent investigators who were blind to the histopathological features, and patient data. Scores were determined by combining the proportion of positively stained tumor cells: 0 , no positive tumor cells; $1,<10 \%$ positive tumor cells; $2,10-50 \%$ positive tumor cells; and $3,>50 \%$ positive tumor cells. The intensity of staining was determined as follows: 0 , no staining; 1 , weak staining/light yellow; 2 , moderate staining/yellowish brown; and 3, strong staining/brown. The staining index was calculated as the product of the proportion of positive cells and the staining intensity score. Using this method of assessment, the protein expression was evaluated by determining the staining index $(0,1,2,3,4,6,9)$. Cut-off values were chosen based on heterogeneity of the log-rank test score with respect to overall survival. The optimal cut-off value was determined: A staining index score $\geq 6$ was used to define tumors with high PRR11 expression; and a score $\leq 4$ indicated low PRR11 expression (12).

IHC was also performed on tumor lesions and normal tissues to measure protein expression in using an AxioVision Rel.4.6 computerized image analysis system and an automatic measurement program (Carl Zeiss AG, Oberkochen, Germany). Specifically, stained sections were evaluated using a light microscope at magnification, x200. A total of 10 representative staining fields of each section were analyzed to verify the mean optical density (MOD), which represents the strength of staining signal (number of positive pixels) (13).

Statistical analysis. Data collection and statistical analysis were performed using SPSS17.0 software (SPSS Inc., Chicago, IL, USA). Differences in PRR11 expression were compared using a student's t-test for comparisons between two groups or one-way analysis of variance with Newman Keul's multiple comparison test for comparisons between $\geq 2$ groups. The $\chi^{2}$ test and Fisher's exact test were used to analyze the association between PRR11 expression, and clinicopathological characteristics. MOD data were statistically analyzed using an unpaired Student's t-test to compare the average MOD difference between different groups of tissues (13). Survival curves were plotted using the Kaplan-Meier method and compared with the log-rank test. The significance of survival variables was 
A

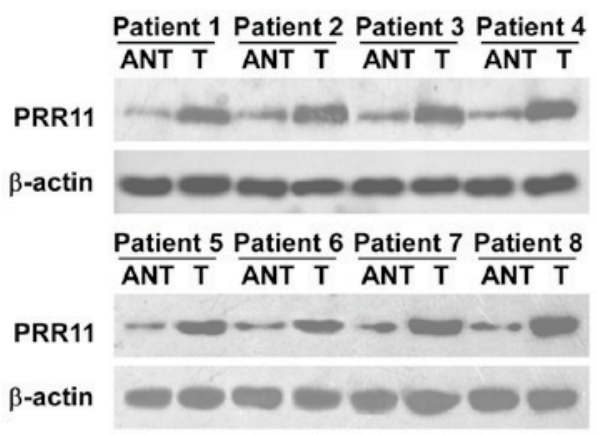

B

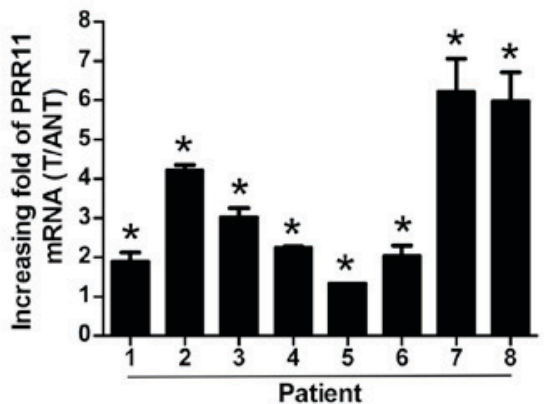

C

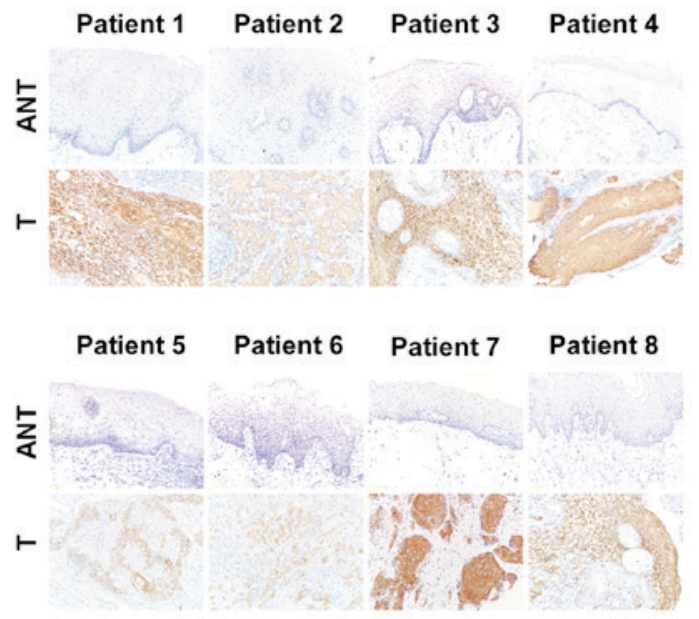

Figure 2. Overexpression of PRR11 in TSCC tissues. Expression of PRR11 at mRNA and protein levels in 8 pairs of T, and ANT from the same patient as determined by (A) western blotting, (B) quantitative polymerase chain reaction analysis and (C) immunohistochemistry (magnification, $\mathrm{x} 200$ ). ${ }^{\mathrm{P}} \mathrm{P}<0.05$ vs. control (Student's t-test). PRR11, proline rich 11; TSCC, tongue squamous cell carcinoma; T, TSCC tissues; ANT, equivalent non-cancerous tissues.

A

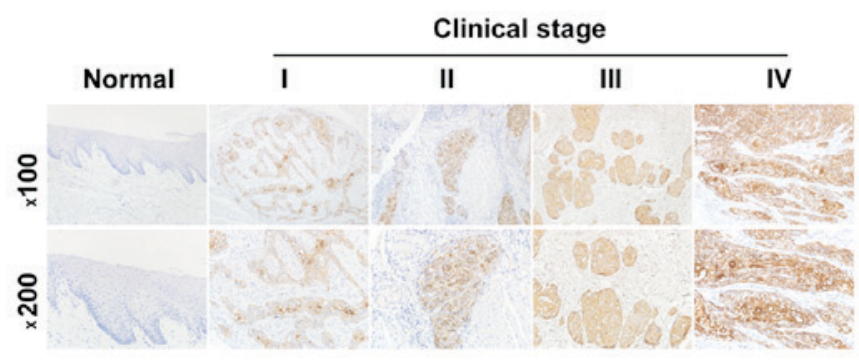

B

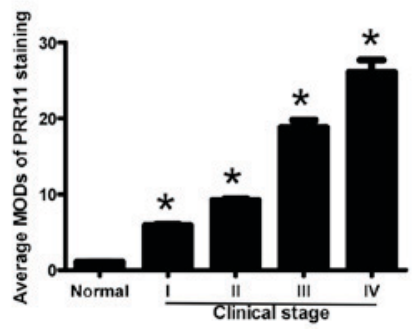

Figure 3. Immunohistochemistry of PRR11 overexpression in archived paraffin-embedded TSCC tissue sections. (A) Representative immunohistochemistry images in normal human oral mucosal tissues and TSCC tissues from different clinical stages (magnifications, x100 and x200). (B) Statistical analysis of average PRR11 MOD staining between normal human oral mucosal tissues and TSCC specimens from different clinical stages. ${ }^{*}<0.05$ vs. normal tissue, using one-way analysis of variance with Student Newman Keul's test. PRR11, proline rich 11; TSCC, tongue squamous cell carcinoma; MOD, mean optical density.

analyzed using univariate and multivariate Cox's regression analysis. $\mathrm{P}<0.05$ (two-tailed) was considered to indicate a statistically significant difference.

\section{Results}

PRR11 is overexpressed in TSCC tissues and is associated with patient survival. PRR11 transcription was examined in an independent TCGA cohort and a significantly higher expression was observed in TSCC tissues compared with non-cancerous tongue tissue ( $\mathrm{P}=0.004$; Fig. 1A). PRR11 was identified to be significantly upregulated at the mRNA level in 12 human TSCC tissues compared with the equivalent non-cancerous tissues ( $\mathrm{P}=0.002$; Fig. 1B). Additionally, assessment of patient survival using Kaplan-Meier analysis and log-rank test indicated an inverse correlation between 
Table II. Association between clinicopathological characteristics and PRR11 expression in patients with tongue squamous cell carcinoma.

\begin{tabular}{|c|c|c|c|c|c|}
\hline \multirow[b]{2}{*}{ Variable } & \multirow[b]{2}{*}{ Total } & \multicolumn{2}{|c|}{ PRR11 } & \multirow[b]{2}{*}{$\chi^{2}$ test $\mathrm{P}$-value } & \multirow{2}{*}{$\begin{array}{l}\text { Fisher's exact } \\
\text { test P-value }\end{array}$} \\
\hline & & Low $(\%)$ & High (\%) & & \\
\hline \multicolumn{6}{|l|}{ Age, years } \\
\hline$\geq 54$ & 36 & $13(36.1)$ & $23(63.9)$ & 0.808 & 0.809 \\
\hline$<54$ & 36 & $14(38.9)$ & $22(61.1)$ & & \\
\hline \multicolumn{6}{|l|}{ Sex } \\
\hline Male & 38 & $13(34.2)$ & $25(65.8)$ & 0.542 & 0.545 \\
\hline Female & 34 & $14(41.2)$ & $20(58.8)$ & & \\
\hline \multicolumn{6}{|l|}{ Clinical stage } \\
\hline $\mathrm{I}-\mathrm{II}$ & 38 & $22(57.9)$ & $16(42.1)$ & $<0.001$ & $<0.001$ \\
\hline III-IV & 34 & $5(14.7)$ & $29(85.3)$ & & \\
\hline \multicolumn{6}{|l|}{$\mathrm{T}$ classification } \\
\hline $\mathrm{T} 1-\mathrm{T} 2$ & 62 & $27(43.5)$ & $35(56.5)$ & 0.008 & 0.009 \\
\hline $\mathrm{T} 3-\mathrm{T} 4$ & 10 & $0(0)$ & $10(100)$ & & \\
\hline \multicolumn{6}{|l|}{$\mathrm{N}$ classification } \\
\hline N0 & 46 & $22(47.8)$ & $24(52.2)$ & 0.016 & 0.017 \\
\hline N1-N2 & 26 & $5(19.2)$ & $21(80.8)$ & & \\
\hline \multicolumn{6}{|l|}{ Grade (differentiation) } \\
\hline Well & 40 & $17(42.5)$ & $23(57.5)$ & 0.327 & 0.331 \\
\hline Moderate and poor & 32 & $10(31.3)$ & $22(68.7)$ & & \\
\hline \multicolumn{6}{|l|}{ Vital status } \\
\hline Alive & 42 & $21(50.0)$ & $21(50.0)$ & 0.010 & 0.010 \\
\hline Succumbed & 30 & $6(20.0)$ & $24(80.0)$ & & \\
\hline
\end{tabular}

PRR11, proline rich 11; $\mathrm{T}$, tumor; $\mathrm{N}$, node.

PRR11 expression and overall survival time of patients with $\operatorname{TSCC}(\mathrm{P}=0.01$; Fig. 1C).

PRR11 is upregulated in TSCC tissues and is associated with TSCC progression. To verify the results of the TCGA analysis, 8 TSCC tissues and their equivalent noncancerous counterparts were subjected to IHC, western blotting, and RT-qPCR analysis (Fig. 2). PRR11 was markedly upregulated at the protein level in all 8 human TSCC tissues according to western blotting (Fig. 2A) and IHC (Fig. 2C) analyses. In addition, PRR11 mRNA levels, as measured by the tumor/normal tissue ratio, were between 1.9-6.2-fold higher in TSCC tissues compared with their equivalent noncancerous tissues (Fig. 2B).

To additionally explore the prevalence of PRR11 upregulation in TSCC, 72 paraffin-embedded archived TSCC tissues and 5 normal human oral mucosal tissues were subjected to IHC. High levels of PRR11 expression were observed in areas containing primary TSCC cells, while PRR11 was undetectable or only marginally detectable in normal human oral mucosal tissues and equivalent noncancerous tissues (Fig. 3A). Quantitative analysis indicated that the average MOD of PRR11 staining in clinical stage I-IV primary tumors was significantly higher compared with in normal human oral mucosal tissues $(\mathrm{P}<0.05)$, and significantly increased with a progression of tumor stage from I to IV $(\mathrm{P}<0.05$; Fig. $3 \mathrm{~B})$.
Taken together, these results clearly demonstrated that PRR11 expression was elevated in TSCC and was associated with TSCC progression.

PRR11 expression was identified to be significantly associated with clinical stage $(\mathrm{P}<0.001), \mathrm{T}$ classification $(\mathrm{P}=0.009), \mathrm{N}$ classification $(\mathrm{P}=0.017)$ and vital status $(\mathrm{P}=0.010)$, but not with any other clinicopathological features, including age, sex, and differentiation grade (Table II). In conclusion, these data suggest that the upregulation of PRR11 is associated with clinical stage, $\mathrm{T}, \mathrm{N}$ classification and vital status, which additionally support the hypothesis that the overexpression of PRR11 is associated with TSCC progression.

Elevated PRR11 expression is associated with poor prognosis in patients with TSCC. To assess the clinical significance of elevated PRR11 expression in patients with TSCC, survival rates were analyzed using 5-year follow-up data. The 5-year cumulative survival rates of patients with higher and lower PRR11 expression were 36.8 , and $83.8 \%$, respectively. Kaplan-Meier analysis indicated that high PRR11 expression was associated with shorter overall survival time $(\mathrm{P}<0.001$; Fig. 4A). In addition, the prognostic value of PRR11 expression was assessed by separating patients according to pathologic primary tumor $(\mathrm{pT}) /$ pathologic regional lymph nodes $(\mathrm{pN})$ status, clinical stage and differentiation. Upregulation of 
Table III. Univariate and multivariate analyses of prognostic parameters in patients with tongue squamous cell carcinoma assessed using Cox regression analysis.

Univariate analysis $\quad$ Multivariate analysis

Variable

\begin{tabular}{cc}
\hline \multicolumn{1}{c}{ HR $(95 \% \mathrm{CI})$} & P-value \\
$1.460(0.702-3.035)$ & 0.311 \\
$1.057(0.516-2.169)$ & 0.879 \\
$2.324(1.113-4.852)$ & 0.025 \\
$1.532(0.581-4.039)$ & 0.389 \\
$2.033(0.989-4.176)$ & 0.054 \\
$1.590(0.776-3.260)$ & 0.206 \\
$5.523(1.977-15.427)$ & 0.001
\end{tabular}

0.001

$5.454(1.821-16.337)$

0.002

HR, hazard ratio; $95 \% \mathrm{CI}, 95 \%$ confidence interval; $\mathrm{T}$, tumor; $\mathrm{N}$, node.

A

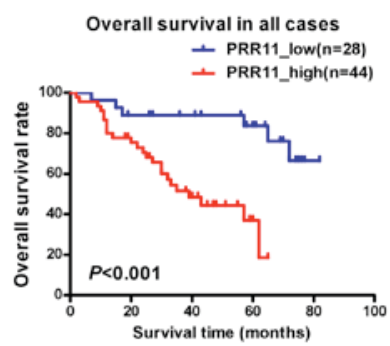

D

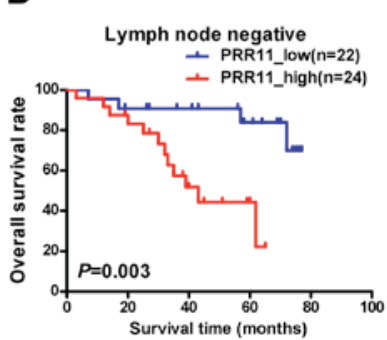

B

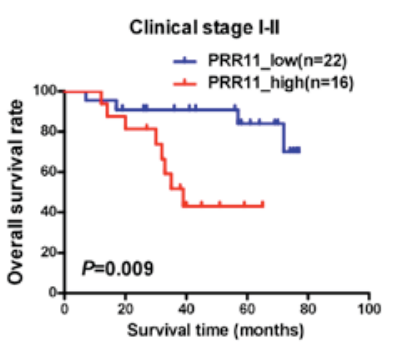

C

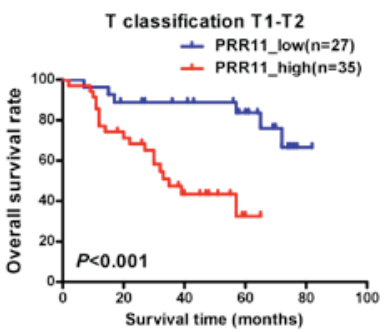

E

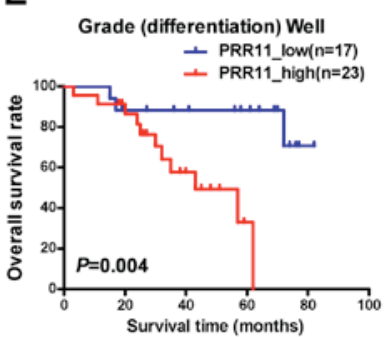

Figure 4. Kaplan-Meier analysis of overall survival in patients with TSCC based on PRR11 expression. (A) OS rates of all patients with high vs. low PRR11 expression. (B) OS rate of patients with stage I-II cancer with high vs. low PRR11 expression. (C) OS rates of patients with T1-T2 grade tumors with high vs low PRR11 expression. (D) OS rates of patients with negative lymph node metastasis with high vs. low PRR11 expression. (E) OS rates of patients with well-differentiated tumors with high vs. low PRR11 expression. P-values were calculated using log-rank tests. TSCC, tongue squamous cell carcinoma; PRR11, proline rich 11; OS, overall survival,

PRR11 was a strong inverse prognostic factor for patients with TSCC in clinical stages I-II (early stage; P=0.009; Fig. 4B). Similarly, patients with higher PRR11 expression demonstrated a significantly shorter survival time (pT1-2, P<0.001, Fig. 4C; lymph node metastasis negative, $\mathrm{P}=0.003$, Fig. 4D; well differentiated, $\mathrm{P}=0.004$, Fig. 4E). However, no statistically significant differences were identified between PRR11 expression and survival time in subsets of clinical stage III-IV, pT3-4, pN1-2, and moderate to poor differentiation, which may reflect the limited number of patients recruited in each subset.

Univariate survival analysis demonstrated that PRR11 expression was significantly associated with poorer overall survival [hazard ratio (HR), 5.523; 95\% confidence interval (CI), 1.977-15.427; $\mathrm{P}=0.001$; Table III]. Multivariate Cox regression analysis revealed that PRR11 expression was an independent prognostic factor for the overall survival of patients with TSCC (HR, 5.454; 95\% CI, 1.821-16.337; P=0.002; Table III). Taken together, these results indicate that PRR11 may be a useful prognostic factor in patients with TSCC.

\section{Discussion}

Previous studies demonstrated that PRR11 overexpression is associated with cancer development and progression in several tumor types (5,8-10). However, the role of PRR11 in TSCC has not been addressed. In the present study, it was identified that PRR11 expression was significantly increased in TSCC tissues compared with non-tumorous oral mucosal 
tissues, and PRR11 overexpression was also associated with tumor stage. Univariate and multivariate Cox regression analyses suggested that PRR11 was an independent predictor for the prognosis of patients with TSCC. Although the present study clarifies the pattern of PRR11 expression and potential clinical significance in TSCC, the potential functions, and exact mechanisms of PRR11 overexpression remain unclear.

It has been demonstrated that PRR11 contains two proline-rich motifs and one zinc-finger domain (8). Proline-rich motifs bind SH3 domains and mediate protein-protein interactions involved in cellular signaling events (14), while zinc-finger domains are known to bind double stranded DNA, and modulate gene transcription (15). Additionally, in lung, breast and numerous types of digestive system cancer, silencing of PRR11 expression induced S-phase arrest, and inhibited cell proliferation, migration, invasion and particularly tumor growth $(5,8,10,16,17)$. However, forced expression of PRR11 inhibited cellular proliferation, and was accompanied by premature chromatin condensation in lung cancer cells (18). This discrepancy suggests that PRR11 may cooperate with other tumor-associated proteins in order to exert its tumor-promoting activity. Additional studies are required to examine this hypothesis.

Lung cancer-associated genes dehydrogenase/reductase 2 (DHRS2), erythrocyte membrane protein band 4.1 like 3 (EPB41L3), cyclin A1 (CCNA1), mitogen-activated protein kinase kinase kinase kinase $4(M A P 4 K 4)$, nuclear factor I B $(N F I B)$ and ribonucleotide reductase catalytic subunit M1 $(R R M 1)$ are deregulated following PRR11 knockdown $(8,16)$. Of these, DHRS2, CCNA1, MAP4K4 and RRM1 are important regulators of cell cycle progression, while CCNA1, $M A P 4 K 4, N F I B$, and EPB $11 L 3$ are involved in tumorigenesis (19-22). In particular, several studies identified that MAP4K4 and EPB41L3 are involved in invasiveness and/or metastasis $(23,24)$. These data indicate that PRR11 may serve a potential role in proliferation, tumorigenesis, invasiveness and/or metastasis. Additionally, in breast cancer, PRR11 depletion reduces the expression of epithelial-mesenchymal transition (EMT)-associated transcription factors snail family transcriptional repressor (SNAI) 1, SNAI2, zinc finger-box-binding homeobox (ZEB) 1 and ZEB2 (9). These are members of the zinc-finger transcription factor family, and are direct repressors of epithelial-cadherin transcription and essential mediators of EMT $(25,26)$. PRR11 may therefore be involved in proliferation, migration, invasion and tumorigenesis by regulating the expression of these, and other genes. However, the molecular mechanisms involved in the association with TSCC patient survival warrant additional investigation.

Due to its unusual histological makeup (rich lymphatic network and highly muscularized structure), the tongue is poorly equipped to protect itself from invasion and metastasis, and TSCC is more frequently associated with metastasis to draining lymph nodes compared with any other cancer of the oral cavity $(25,26)$. As nodal metastasis in the neck is an important prognostic factor, patients with TSCC exhibit a significantly poorer prognosis compared with those patients with cancer in other sites of the oral cavity (27). The clinical course of TSCC is also unpredictable, due to the relatively high rate of occult metastasis in patients presenting with a very small primary tumor without clinical evidence of metastatic disease (28). The use of neck dissection in the surgical management of clinical stage I-II TSCC has been a source of debate for this reason (29).

The results of the present study indicated that patients with high levels of PRR11 expression exhibited a shorter survival time, and PRR11 expression was also associated with regional draining lymph nodes metastasis. PRR11 may therefore be a useful prognostic marker in patients with TSCC, and may indicate whether the use of neck dissection in clinical stages I-II is a sensible option in the absence of TNM staging information.

\section{Acknowledgements}

The present study was supported by the National Natural Science Foundation of China (grant nos., 81272948 and 81201548).

\section{References}

1. Chen YK, Huang HC, Lin LM and Lin CC: Primary oral squamous cell carcinoma: An analysis of 703 cases in southern Taiwan. Oral Oncol 35: 173-179, 1999.

2. Sano D and Myers JN: Metastasis of squamous cell carcinoma of the oral tongue. Cancer Metastasis Rev 26: 645-662, 2007.

3. Greene FL, Page DL, Fleming ID, et al: AJCC Cancer Staging Hhandbook. 6th edition. New York, Springer-Verlag, pp35-46, 2002.

4. Ota T, Suzuki Y, Nishikawa T, Otsuki T, Sugiyama T, Irie R, Wakamatsu A, Hayashi K, Sato H, Nagai K, et al: Complete sequencing and characterization of 21,243 full-length human cDNAs. Nat Genet 36: 40-45, 2004.

5. Chen Y, Cha Z, Fang W, Qian B, Yu W, Li W, Yu G and Gao Y: The prognostic potential and oncogenic effects of PRR11 expression in hilar cholangiocarcinoma. Oncotarget 6: 20419-20433, 2015.

6. Tai YC, Domchek S, Parmigiani G and Chen S: Breast cancer risk among male BRCA1 and BRCA2 mutation carriers. J Nat Cancer Inst 99: 1811-1814, 2007.

7. Yan J, Jiang J, Lim CA, Wu Q, Ng HH and Chin KC: BLIMP1 regulates cell growth through repression of p53 transcription. Proc Natl Acad Sci USA 104: 1841-1846, 2007.

8. Ji Y, Xie M, Lan H, Zhang Y, Long Y, Weng H, Li D, Cai W, Zhu H, Niu Y, et al: PRR11 is a novel gene implicated in cell cycle progression and lung cancer. Int J Biochem Cell Biol 45: 645-656, 2013.

9. Zhou F, Liu H, Zhang X, Shen Y, Zheng D, Zhang A, Lai Y and Li H: Proline-rich protein 11 regulates epithelial-to-mesenchymal transition to promote breast cancer cell invasion. Int J Clin Exp Pathol 7: 8692-8699, 2014.

10. Song Z, Liu W, Xiao Y, Zhang M, Luo Y, Yuan W, Xu Y, Yu G and $\mathrm{Hu}$ Y: PRR11 is a prognostic marker and potential oncogene in patients with gastric cancer. PLoS One 10: e128943, 2015.

11. Livak KJ and Schmittgen TD: Analysis of relative gene expression data using real time quantitative PCR and the 2(-Delta Delta C(T)) method. Methods 25: 402-408, 2001.

12. Song L, Gong H, Lin C, Wang C, Liu L, Wu J, Li M and Li J: Flotillin-1 promotes tumor necrosis factor- $\alpha$ receptor signaling and activation of $\mathrm{NF}-\kappa \mathrm{B}$ in esophageal squamous cell carcinoma cells. Gastroenterology 143: 995-1005.e12, 2012.

13. Li J, Yu L, Zhang H, Wu J, Yuan J, Li X and Li M: Down-regulation of pescadillo inhibits proliferation and tumorigenicity of breast cancer cells. Cancer Sci 100: 2255-2260, 2009.

14. Ball LJ, Kühne R, Schneider-Mergener J and Oschkinat H: Recognition of proline-rich motifs by protein-protein-interaction domains. Angew Chem Int Ed Engl 44: 2852-2869, 2005.

15. Sera T: Zinc-finger-based artificial transcription factors and their applications. Adv Drug Deliv Rev 61: 513-526, 2009.

16. Zhao Q: RNAi-mediated silencing of praline-rich gene causes growth reduction in human lung cancer cells. Int J Clin Exp Pathol 8: 1760-1767, 2015.

17. Wang Y, Zhang Y, Zhang C, Weng H, Li Y, Cai W, Xie M, Long Y, Ai Q, Liu Z, et al: The gene pair PRR11 and SKA2 shares a NF-Y-regulated bidirectional promoter and contributes to lung cancer development. Biochim Biophys Acta 1849: 1133-1144, 2015. 
18. Zhang C, Zhang Y, Li Y, Zhu H, Wang Y, Cai W, Zhu J, Ozaki T and $\mathrm{Bu}$ Y: PRR11 regulates late-S to $\mathrm{G} 2 / \mathrm{M}$ phase progression and induces premature chromatin condensation (PCC). Biochem Biophys Res Commun 458: 501-508, 2015.

19. Dooley AL, Winslow MM, Chiang DY, Banerji S, Stransky N, Dayton TL, Snyder EL, Senna S, Whittaker CA, Bronson RT, et al: Nuclear factor I/B is an oncogene in small cell lung cancer. Genes Dev 25: 1470-1475, 2011.

20. Liu AW, Cai J, Zhao XL, Jiang TH, He TF, Fu HQ, Zhu MH and Zhang SH: ShRNA-targeted MAP4K4 inhibits hepatocellular carcinoma growth. Clin Cancer Res 17: 710-720, 2011.

21. Malumbres $\mathbf{M}$ and Carnero A: Cell cycle deregulation: A common motif in cancer. Prog Cell Cycle Res 5: 5-18, 2003.

22. Yageta M, Kuramochi M, Masuda M, Fukami T, Fukuhara H, Maruyama T, Shibuya M and Murakami Y: Direct association of TSLC1 and DAL-1, two distinct tumor suppressor proteins in lung cancer. Cancer Res 62: 5129-5133, 2002.

23. Bernkopf DB and Williams ED: Potential role of EPB41L3 (protein 4.1B/Dal-1) as a target for treatment of advanced prostate cancer. Expert Opin Ther Targets 12: 845-853, 2008.

24. Collins CS, Hong J, Sapinoso L, Zhou Y, Liu Z, Micklash K, Schultz PG and Hampton GM: A small interfering RNA screen for modulators of tumor cell motility identifies MAP4K4 as a promigratory kinase. Proc Natl Acad Sci USA 103: 3775-3780, 2006.

25. Cano A, Pérez-Moreno MA, Rodrigo I, Locascio A, Blanco MJ, del Barrio MG, Portillo F and Nieto MA: The transcription factor snail controls epithelial-mesenchymal transitions by repressing E-cadherin expression. Nat Cell Biol 2: 76-83, 2000.
26. Lo HW, Hsu SC, Xia W, Cao X, Shih JY, Wei Y, Abbruzzese JL, Hortobagyi GN and Hung MC: Epidermal growth factor receptor cooperates with signal transducer and activator of transcription 3 to induce epithelial-mesenchymal transition in cancer cells via up-regulation of TWIST gene expression. Cancer Res 67: 9066-9076, 2007.

27. Rusthoven K, Ballonoff A, Raben D and Chen C: Poor prognosis in patients with stage I and II oral tongue squamous cell carcinoma. Cancer 112: 345-351, 2008.

28. Byers RM, El-Naggar AK, Lee YY, Rao B, Fornage B, Terry NH, Sample D, Hankins P, Smith TL and Wolf PJ: Can we detect or predict the presence of occult nodal metastases in patients with squamous carcinoma of the oral tongue? Head Neck 20: 138-144, 1998.

29. D'Cruz AK, Siddachari RC, Walvekar RR, Pantvaidya GH, Chaukar DA, Deshpande MS, Pai PS and Chaturvedi P: Elective neck dissection for the management of the N0 neck in early cancer of the oral tongue: Need for a randomized controlled trial. Head Neck 31: 618-624, 2009. 